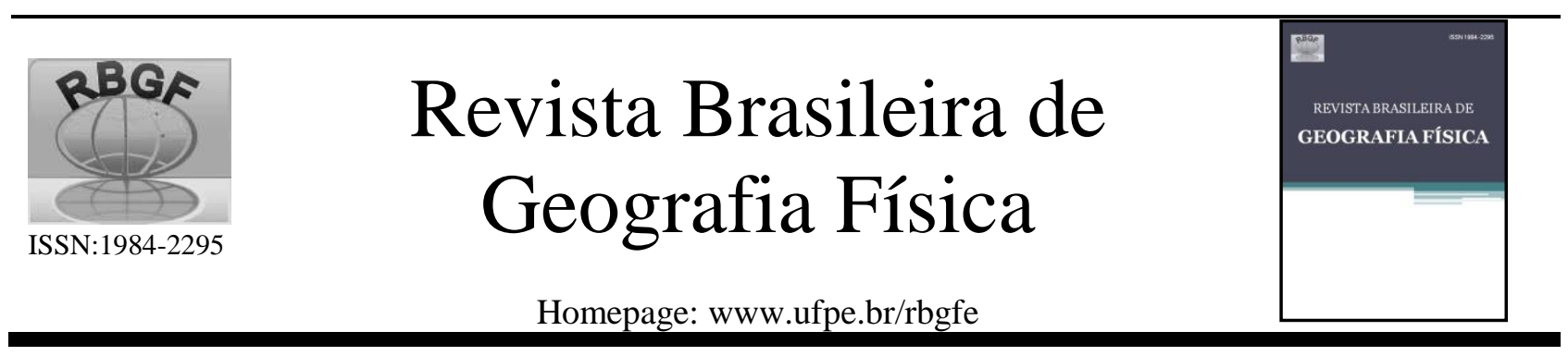

\title{
Aspectos da paisagem e fatores socioeconômicos nos casos de dengue na cidade de Porto Alegre, RS
}

\author{
Jessica Mazutti Penso-Campos ${ }^{1}$, Eliane Fraga ${ }^{2}$, Eduardo Caldas ${ }^{3}$, Jussara Alves Pinheiro Sommer ${ }^{4}$, Eduardo \\ Périco 5
}

\begin{abstract}
${ }^{1}$ Mestra e Doutoranda em Ambiente e Desenvolvimento na Universidade do Vale do Taquari - UNIVATES, Lajeado, RS. jessica.m.penso@gmail.com (Autor correspondente). ${ }^{2}$ Professora doutora do Programa de Pós-Graduação em Promoção da Saúde (PPGProsaúde) da Universidade Luterana do Brasil - ULBRA, Canoas, RS.

elianefraga3@ hotmail.com. ${ }^{3}$ Biólogo da Universidade Luterana do Brasil - ULBRA, Canoas, RS.

eduardo_caldas@ @otmail.com ${ }^{4}$ Professora doutora do Curso de Geografia da Universidade Luterana do Brasil ULBRA, Canoas, RS. japsommer@ sinos.net ${ }^{5}$ Professor doutor do Programa de Pós-Graduação em Ambiente e Desenvolvimento (PPGAD) da Universidade do Vale do Taquari - UNIVATES, Lajeado, RS. perico@ univates.br.
\end{abstract}

Artigo recebido em 14/04/2018 e aceito em 27/07/2018

\begin{abstract}
R E S U M O
A dengue é uma doença causada por um arbovírus transmitido pelo mosquito Aedes aegypti. Nas áreas urbanas, a falta de planejamento ambiental e a desigualdade das condições socioeconômicas favoreceram o aumento dos casos da doença, mesmo em locais com clima mais variável, como o sul do Brasil. Em 2001, houve a introdução de A. aegypti na cidade de Porto Alegre, RS. Objetivou-se correlacionar casos de dengue autóctones com variáveis socioeconômicas e ambientais em Porto Alegre. No período de janeiro a julho de 2013 monitoraram-se 712 armadilhas para mosquitos adultos, em 22 bairros da cidade. Para análise de variáveis ambientais, recorreu-se aos dados de cobertura e uso do solo para cada bairro amostrado, conforme as seguintes classes: cobertura vegetal (árvores, arbustos e herbáceos), água e uso humano (outros usos). Utilizaram-se como variáveis, a cobertura e uso do solo, precipitação, temperatura, renda média e a densidade populacional por bairro. Aplicou-se a análise de regressão múltipla para avaliar a importância de cada fator em relação ao número de fêmea e casos relatados. Os resultados indicam que a precipitação não influenciou significativamente o número de fêmeas coletadas, mas a temperatura tem uma relação significativa. O número de fêmeas coletadas eleva-se com o aumento da temperatura. A análise de regressão múltipla indica que a variável dependente (número de casos) foi significativamente influenciada pelo número de fêmeas $(\mathrm{p}=0,0420)$, a cobertura vegetal $(\mathrm{p}=0,0005)$ e o uso antrópico $(\mathrm{p}=0,0003)$.

Palavras-chave: Cobertura e uso da terra; ArcGis; geoprocessamento; processamento de imagens; vigilância ambiental em saúde.
\end{abstract}

\section{Landscape aspects and socioeconomic factors in the cases of Dengue in the city of Porto Alegre, RS}

\begin{abstract}
A B S T R A C T
The dengue is a disease caused by an arbovirosis transmitted by the mosquito Aedes aegypti. In urban areas, the lack of environmental planning and difference in socioeconomic conditions have favored an increase in the disease cases even in places with milder weather, such as southern Brazil. In 2001, there was the introduction of A. aegypti in the city of Porto Alegre, RS. The aim of the study was to correlate cases of autochthonous dengue with socioeconomic and environmental variables in Porto Alegre. From January to July 2013, 712 traps for adult mosquitoes were monitored in 22 city neighborhoods. For analysis of environmental variables, data of use and land cover were obtained for each sampled neighborhood and separated into the following classes: vegetation cover (trees, shrubs and herbaceous), water and human use (other uses). Besides the land use and cover the influence of rainfall, temperature, average income and population density have been verified. Multiple regression analysis was performed to assess the importance of each factor in the number of reported cases. The results indicated that precipitation did not significantly influence the number of females collected, but the temperature has a significant relationship. The number of females collected increases with increasing temperature. The multiple regression analysis indicates that the dependent variable (number of cases) was significantly influenced by the number of females $(p=0.0420)$, the vegetation cover $(p=0.0005)$ and human use $(p=0.0003)$, but the density and average income.
\end{abstract}


Keywords: Coverage and land use; ArcGis; geoprocessing; image processing; environmental health surveillance.

\section{Introdução}

As alterações no uso e ocupação do solo, causadas principalmente pela ação antrópica, como conversão de áreas naturais para uso agropecuário ou urbano, atuam de diversas formas para o desenvolvimento e disseminação de epidemias. A redução de áreas de vida de organismos vetores e a eliminação de predadores naturais, em conjunto com a fragmentação de habitats, que aproxima o espaço urbano do espaço natural, cria um ambiente propício para as doenças vetoriais. (Brasil, 2006a; Périco e Cemin, 2006).

As epidemias recorrentes de dengue têm como fatores relacionados, principalmente, a proliferação dos vetores, em especial do Aedes aegypti, o acelerado crescimento demográfico, a urbanização, a desacertada organização da infraestrutura urbana, a acumulação de resíduos não orgânicos que servem de criadouros aos vetores, os limites nas fronteiras do conhecimento dos agentes de saúde e da população geral sobre o tema e a negligência coletiva em relação à prevenção da proliferação do vetor (Mendonça et. al., 2009).

O estudo de Almeida e Silva (2017), sobre casos de dengue em João Pessoa, Cabedelo e Bayeux, na Paraíba, realizou análise in loco nos locais com maior tendência à difusão de dengue. A importância de disseminar o conhecimento, através da conscientização da população, fica claro pelas imagens fotográficas que mostram o acúmulo inadequado de resíduos não orgânicos a céu aberto, como por exemplo, de pneus e garrafas, servindo como possíveis criadouros para os vetores, refletindo a negligência da coletividade no que tange a prevenção da doença, permeada pela prática simples da eliminação dos criadouros, principalmente através do destino adequado dos resíduos.

A dengue é uma doença febril aguda que afeta anualmente cerca de 100 milhões de pessoas em todo o mundo (Baroni e Oliveira, 2009), sendo transmitida pelos mosquitos A. aegypti e Aedes albopictus (Tauil, 2001).

A fêmea infectada do mosquito A. aegypti é a principal responsável pela propagação. $\mathrm{O}$ mosquito desenvolve-se principalmente em áreas tropicais e subtropicais devido às condições climáticas e ambientais que beneficiam sua proliferação (Baroni e Oliveira, 2009).

Um dos principais fatores associados à enfermidade é a presença de criadouros para o vetor, dos quais pode-se citar os ferros-velhos, as borracharias, os terrenos baldios e outros locais que tendem a apresentar água parada (Ferreira 2004 apud Flauzino et. al., 2009).

O crescimento populacional, o aumento da desigualdade social e o inadequado ordenamento ambiental favorecem uma alta ocorrência do vetor em áreas urbanas, processo conhecido por domiciliação (Forattini, 1992).

Demais fatores ambientais, como temperaturas elevadas e grande incidência de chuva contribuem de forma significativa para a proliferação do mosquito, juntamente com o aumento do número de casos de dengue (Honório e Oliveira, 2001; Ribeiro et al., 2006).

Outro fator determinante para a proliferação do $A$. aegypti são as condições socioeconômicas da população (Costa e Natal 1998; Mondini e Neto 2007; Flauzino et al., 2009; Almeida e Silva, 2017). Mendonça et. al. (2009) citam que "a intensificação da urbanização decorreu principalmente do avanço da industrialização, que atraiu grandes fluxos de mãode-obra do campo [...] no último meio século a urbanização brasileira caracterizou-se predominantemente por uma dinâmica desordenada e sem infraestrutura adequada, com grandes contingentes populacionais em condições de pobreza e miserabilidade" (p. 259).

A inquietude dos órgãos sanitários acerca do combate à dengue está relacionada ao impacto econômico da doença (Valdés, 2002), assim como pelo grande potencial de evolução para condições graves e letais (Flauzino et. al., 2009).

A Reforma Sanitária Brasileira refletiu a intensa estratégia de mobilização acerca da qualificação das condições de higiene e organização do território, integrando o esforço para a melhoria do planejamento urbano e o reconhecimento sobre a importância do saneamento básico para a saúde pública, principalmente no que tange o controle de vetores a fim de atingir a erradicação das doenças transmissíveis.

Contudo, mesmo diante do impacto positivo que este movimento causou em relação ao controle das doenças, verifica-se a reincidência de epidemias transmissíveis, como a Dengue e outras disseminadas por vetores em comum (Mendonça et. al., 2009).

O A. aegypti é o vetor transmissor da Dengue, Chikungunya, Febre Amarela e Zica Vírus. O Ministério da Saúde declarou situação de Emergência em Saúde Pública de importância Nacional (ESPIN) por alteração do padrão de ocorrência de microcefalias no Brasil, relacionada 
ao Zika Vírus, através da Portaria $\mathrm{n}^{\circ} 1.813$, de 11 de novembro de 2015 (Brasil, 2015).

Nos 18 meses seguintes ocorreram ações intersetoriais, em âmbito nacional, de fortalecimento ao combate do A. aegypti, nas três esferas de gestão do Sistema Único de Saúde (SUS), resultando na redução da ocorrência de microcefalias no Brasil e no encerramento da ESPIN, conforme a Portaria $\mathrm{n}^{\circ} 1.682$, de 30 de julho de 2017 (Brasil, 2017).

No Rio Grande do Sul, entre 2016 a 2017, confirmaram-se, para o conjunto de casos autóctones e importados, 90 casos de Chikungunya e 85 de Zica Vírus ${ }^{1}$. Para o período de 2018, até a semana epidemiológica $\mathrm{n}^{\mathrm{o}} 5$ (28/01/2018 a 03/02/2018), notificaram-se 151 casos de dengue (nenhum autóctone até o momento), 16 casos de Febre de Chikungunya e 9 casos de Febre Amarela, todos os casos notificados continuaram em investigação durante o desenvolvimento deste artigo.

Em relação à Doença Aguda pelo Zika Vírus, em 2018 até semana epidemiológica $\mathrm{n}^{\circ} 3$ (14/01/2018 a 20/01/2018), notificaram-se 131 casos, dos quais 19 (14,5\%) foram confirmados, os demais continuam sendo investigados ${ }^{2}$.

Os primeiros registros de casos autóctones de Dengue no Rio Grande do Sul ocorreram em 2007, na cidade de Giruá, localizada na região noroeste (Baroni e Oliveira, 2009). Os casos da doença tendem a ser notificados com maior tendência entre os meses de novembro a maio, considerando-se o período de sazonalidade da dengue (Rio Grande do Sul, 2017).

Em relação ao ano de 2018, os dados preliminares disponíveis no SINAN, durante o desenvolvimento do presente estudo, que tangem até a semana epidemiológica de número 05 (28/01/2018 a 03/02/2018), apontam para 151 casos notificados, nenhum deles confirmados e/ou autóctone de Dengue no estado do Rio Grande do Sul (SINAN, 2018).

No que tange a série histórica de 2010 a 2017, até o período com dados disponíveis, registrou-se para o ano de 2017, o menor número de confirmações da doença ${ }^{1}$.

\footnotetext{
${ }^{1}$ Informativo Epidemiológico Dengue, Chikungunya e Zika Vírus de Novembro de 2017. Rio Grande do Sul. Disponível em: http://www.saude.rs.gov.br/upload/arquivos/carga2017 1136/06093648-informativo-epidemiologico-denguechik-e-zika-se-43-2017.pdf Acesso em: 09 de Março de 2018.

2 Informativo Epidemiológico de Arboviroses de Fevereiro de 2018. Rio Grande do Sul. Disponível em: http://www.saude.rs.gov.br/upload/arquivos/carga2018
}

Em 2001 houve a introdução da espécie $A$. aegypti na cidade de Porto Alegre, sendo notificados casos importados no verão. Somente em 2010 foram confirmados os primeiros casos autóctones da doença na cidade, sendo todos os casos do bairro Jardim Carvalho. Em 2011 a cidade teve 12 casos autóctones da doença distribuídos pelos bairros Farroupilha, Santo Antônio e Azenha. Em 2012 a cidade não teve nenhum registro de caso autóctone.

Em abril de 2013 o LIRAa (Levantamento de Índice Rápido de A. aegypti) indicou que o nível de infestação do mosquito da dengue em Porto Alegre aumentou de 4,6\% em janeiro para 5,8\%, sendo que o ideal é que fique em até $1 \%{ }^{3}$.

O objetivo do presente trabalho foi analisar a relação entre a distribuição espacial de fêmeas do A. aegypti e dos casos de dengue com os aspectos da paisagem, tendo como indicador o uso e cobertura do solo, e com as variáveis socioeconômicas da população, em 22 bairros da cidade de Porto Alegre, Rio Grande do Sul.

\section{Metodologia}

Realizou-se o estudo na cidade de Porto Alegre, RS (Figura 1), que apresenta uma área territorial de 496,682 $\mathrm{km}^{2}$, integrando esgotamento sanitário de $93 \%$, arborização de vias públicas de $82,7 \%$, urbanização de vias públicas de $69,4 \%$, população estimada no ano de 2017 em 1.484 .941 habitantes, com tendência de pequena elevação em relação à população censitária de 2010, que apontava para 1.409.351 habitantes, com densidade demográfica de 2.837,53 hab/ $\mathrm{km}^{2}$.

A carga tributária do Produto Interno Bruto per capita no período de 2015 expresso em $\mathrm{R} \$$ 46.122,79 e Índice de Desenvolvimento Humano Municipal (IDHm), em 2010, estimado em 0,805, liderando o ranking no Estado (IBGE, 2017).

Porto Alegre é a capital mais meridional do país $\left(30^{\circ} 01^{\prime} 42^{\prime \prime} \mathrm{S}\right.$ e $\left.51^{\circ} 13^{\prime} 42^{\prime \prime} \mathrm{W}\right)$, com altitude média de $10 \mathrm{~m}$ e máxima de $311 \mathrm{~m}$. Devida a sua posição está sujeita aos avanços constantes de massas de ar polares, estimando-se em $6^{\circ} \mathrm{C}$ a temperatura da isoterma ${ }^{4}$.

0202/09160220-informativo-epidemiologico-denguese05-2018-3.pdf Acesso em: 09 de Março de 2018.

3 Porto Alegre. Secretaria Municipal da Saúde. Disponível em: http://www2.portoalegre.rs.gov.br/dengue/default.php? p_secao=11 Acesso em: 15 de setembro de 2013.

${ }^{4}$ Instituto Nacional de Meteorologia (INMET) (2017). Disponível em: http://www.inmet.gov.br/portal/ Acesso em 09 de Março de 2018. 
O clima é considerado subtropical úmido (Cfa) e, a pluviosidade, umidade e temperatura médias anuais são, respectivamente, $112 \mathrm{~mm}, 76 \%$ e $19,5^{\circ} \mathrm{C}$ (IBGE, 2017).

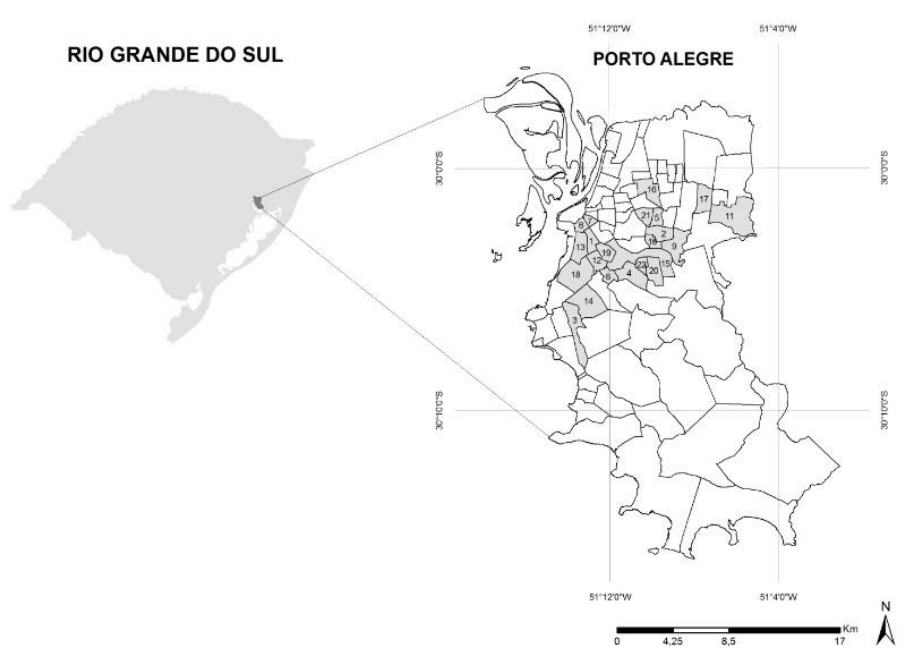

Figura 1. Localização da cidade de Porto Alegre, RS, destaque aos bairros amostrados.

Em 2009 o Ministério da Saúde apresentou as Diretrizes Nacionais para Prevenção e Controle de Epidemias de Dengue, visando prevenir a mortalidade e os processos epidêmicos relacionados à doença, através da organização de fluxogramas (Figura 2) e informações em saúde que servem de embasamento aos gestores e profissionais de saúde, acerca da organização da rede de atenção à saúde no cuidado ao paciente acometido pela doença, o fortalecimento das ações de vigilância em saúde, a fim de garantir a investigação, a notificação e o monitoramento de casos da doença e dos locais vulneráveis à instauração de criadouros do vetor e o fortalecimento de ações articuladas intersetorialmente (Brasil, 2009)

A Figura 3 reflete a série histórica da Dengue segundo os dados do Sistema de Informação de Agravos de Notificação (SINAN).

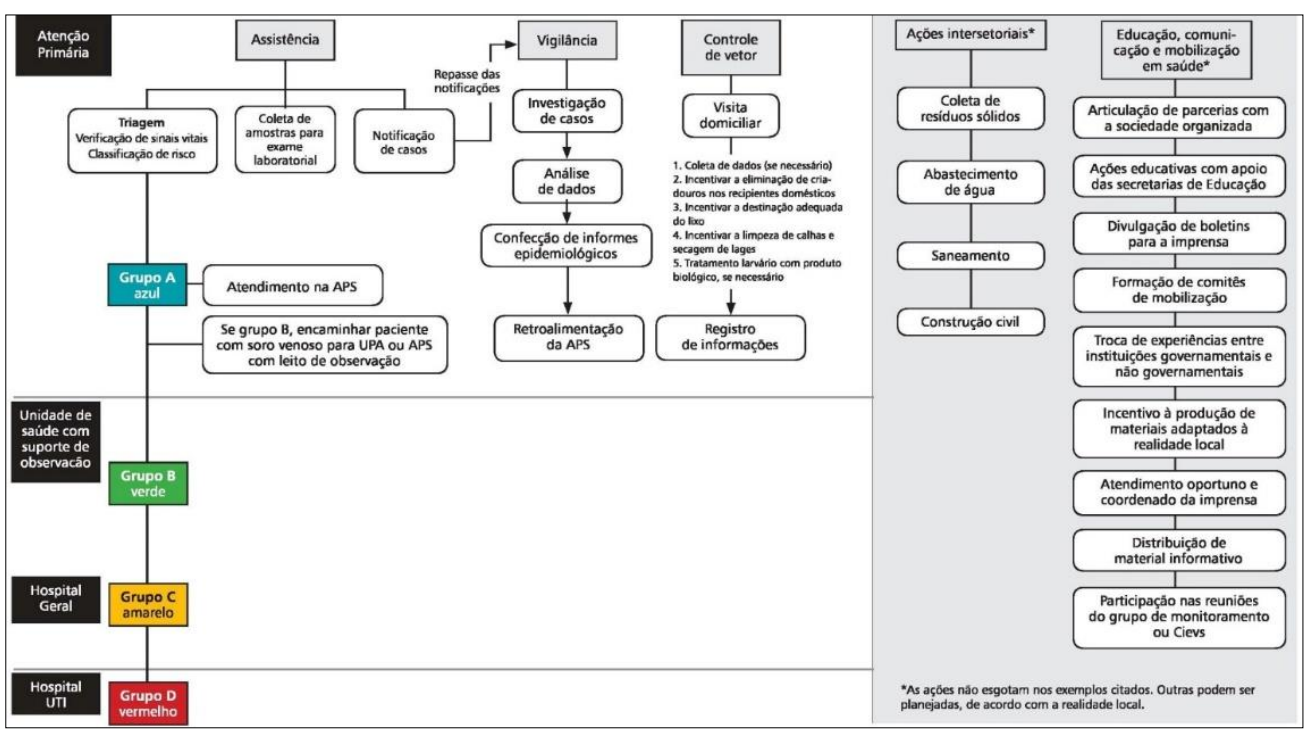

Figura 2. Proposta de fluxograma para o controle da dengue em nível municipal apresentado nas Diretrizes Nacionais para Prevenção e Controle de Epidemias de Dengue (Brasil, 2009, p. 103).

Fonte: Brasil. Ministério da Saúde. Secretaria de Vigilância em Saúde. Departamento de Vigilância Epidemiológica. Diretrizes nacionais para prevenção e controle de epidemias de dengue, Brasília : Ministério da Saúde, 2009, p. 103. Legenda: APS = Atenção Primária à Saúde / Atenção Básica à Saúde; UPA= Unidade de Pronto Atendimento; UTI=Unidade de Terapia Intensiva; Grupo (Classificação de Risco): A= Sem sangramento ou sinais de alarme, B=Com sangramento, $\mathrm{C}=$ com sinais de alarme, $\mathrm{D}=$ com sinais de choque (Brasil, 2009). 


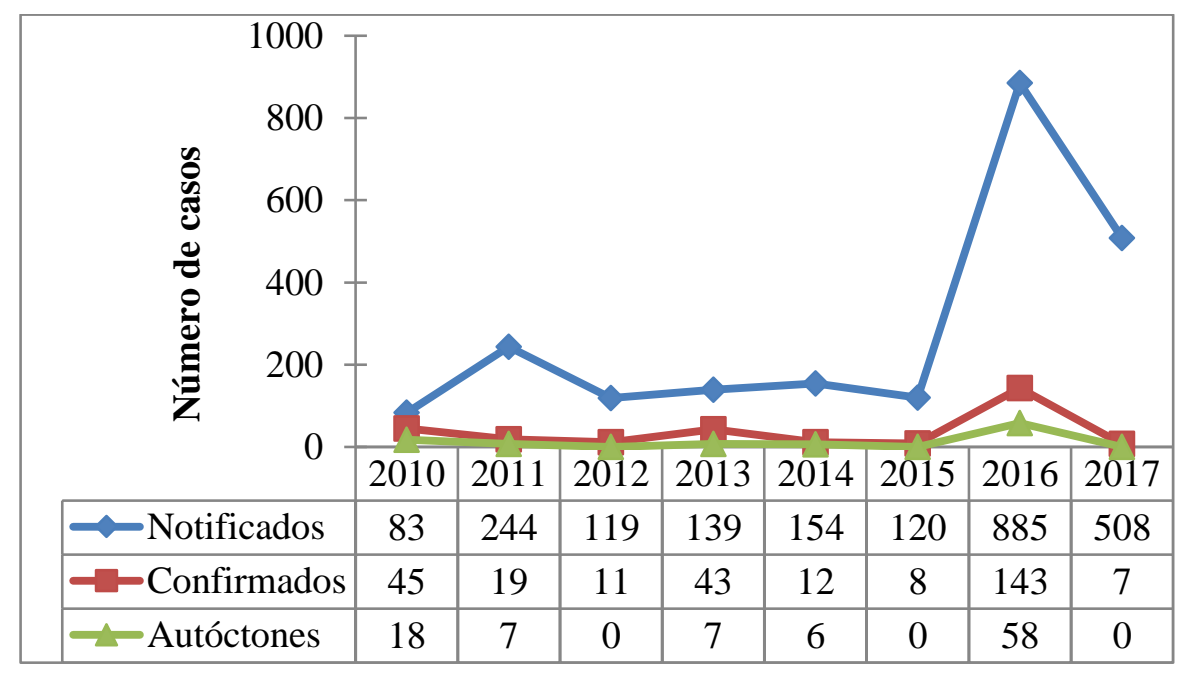

Figura 3. Série histórica do número total de casos de Dengue no Rio Grande do Sul, 2010 a 2016. Fonte: elaborado a partir dos dados do SINAN (2018) e Informativo Epidemiológico ${ }^{2}$ (acima).

As Diretrizes Nacionais para Prevenção e Controle de Epidemias de Dengue (Brasil, 2009) apresentam uma proposta de estrutura hierárquica responsável pelas ações de investigação, controle e monitoramento dos focos de A. aegypti (Figura 4).

Essa estrutura reflete a ação conjunta entre a Atenção Primária à Saúde e a Vigilância em Saúde, mobilizando a participação de Agentes Comunitários de Saúde, Agentes de Controle às Endemias e equipe multidisciplinar, realizando-se como principal atividade a verificação de focos do vetor e o Levantamento de Índice Rápido para Aedes aegypti (LIRAa) (Brasil, 2009).

Em Porto Alegre o LIRAa caracteriza-se como uma das principais estratégias para monitoramento dos focos do $A$. aegypti, onde verifica-se a presença de larvas da espécie, para posteriormente realizar um levantamento com os bairros mais vulneráveis.

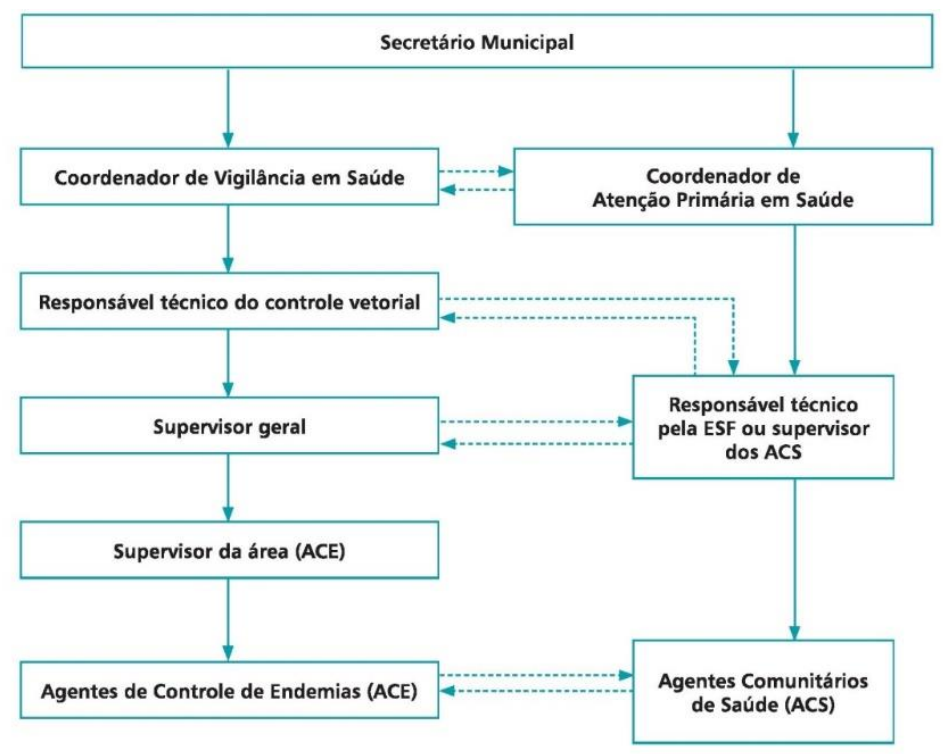

Figura 4. Proposta de fluxograma da estrutura hierárquica para o controle do A. aegypti em nível municipal integrado das Diretrizes Nacionais para Prevenção e Controle de Epidemias de Dengue (Brasil, 2009, p. 60). Fonte: Brasil. Ministério da Saúde. Secretaria de Vigilância em Saúde. Departamento de Vigilância Epidemiológica. Diretrizes nacionais para prevenção e controle de epidemias de dengue, Brasília : Ministério da Saúde, 2009, p. 60. Legenda: ESF: Estratégia Saúde da Família (principal estratégia da Atenção Básica à Saúde para o cuidado integral do sujeito e seu núcleo familiar); ACS: Agente Comunitário de Saúde; ACE: Agente de Controle de Endemias.

O monitoramento com armadilhas para mosquitos adultos foi implantado em 2012 pelo
Controle Operacional da Dengue / Coordenadoria Geral de Vigilância em Saúde (COD/CGVS), em 
contrato com a empresa ECOVEC, que detém tecnologia patenteada para essa armadilha. A armadilha MosquiTRAP versão 2.0 imita um criadouro para as fêmeas adultas do mosquito $A$. aegypti.

O estudo avaliou 712 armadilhas dispersas em 22 bairros da cidade de Porto Alegre visando encontrar fêmeas de A. aegypti. As armadilhas foram instaladas pelo COD/CGVS, de acordo com o critério de vulnerabilidade do bairro para a dengue obtido pelo LIRAa (Levantamento de Índice Rápido do $A$. aegypti).

As armadilhas foram implantadas a $250 \mathrm{~m}$ de distância uma da outra, a fim de estabelecer uma cobertura homogênea nos bairros selecionados e o endereço foi registrado pela sua localização com GPS. O estudo foi realizado de janeiro a julho de 2013.

Para verificar a influência da precipitação e da temperatura máxima por mês amostrado em relação ao número médio de fêmeas amostradas foi aplicada uma análise de regressão múltipla. A diferença mensal entre o número médio de fêmeas foi calculada pelo teste de Kruskal-Wallis e teste de Dunn.

Para a obtenção dos dados de variáveis climáticas foram utilizados os dados disponibilizados pelo INMET. A renda média de cada um dos 22 bairros foi obtida no Instituto Brasileiro de Geografia e Estatística (IBGE) (2017).

Obtiveram-se os dados de uso e cobertura da terra para cada bairro amostrado, categorizandose em: cobertura vegetal (arbóreo, arbustivo e herbáceo), água e uso antrópico (demais usos). Coletaram-se também os dados de renda média e densidade populacional (habitantes $/ \mathrm{Km}^{2}$ ) por bairro e, número de fêmeas e casos autóctones de dengue confirmados.

Realizaram-se duas análises de regressão múltipla para verificar a influência de cada uma das variáveis independentes sobre o número de casos e sobre o número de fêmeas registradas (variáveis dependentes).

Elaboraram-se os mapas no software ArcGis 10.2, com o objetivo de criar um mapa final de suscetibilidade para dengue. O mapa de uso e cobertura do solo foi elaborado com base no diagnóstico ambiental realizado pela Universidade Federal do Rio Grande do Sul ${ }^{5}$.

Para elaboração do mapa de uso do solo, utilizou-se o shapefile do município de Porto Alegre. Nos 22 bairros amostrados aplicou-se a ferramenta Clip para extrair as classes de uso do

5 Hasenack, H., Weber, E. (org.), 2010. Base cartográfica vetorial contínua do Rio Grande do Sul - solo de interesse, divididas em água, arbóreo, arbustivo, herbáceo e uso antrópico, em hectares (ha).

Para os mapas de número de casos, renda média, densidade demográfica, vegetação, uso antrópico, água e número de fêmeas utilizou-se o shapefile do município de Porto Alegre com os bairros de estudo em destaque e com um degradê de cores em cinza.

$\mathrm{Na}$ ferramenta Polygon to Raster selecionou-se o arquivo vetorial dos bairros de Porto Alegre para a geração dos mapas citados anteriormente em formato de imagem (variáveis independentes), com um degradê de cores de cinza. Os mapas gerados não seguiam uma normalidade, dessa forma necessitou-se realizar uma normalização dos seis mapas, utilizando a lógica fuzzy, com a ferramenta Fuzzy Membership, na forma linear, tornando o degradê de cores entre 0 e 1 .

Dentre as variáveis independentes trabalhadas, apenas a vegetação apresentou valor inversamente proporcional ao número de casos, quanto menor a área com cobertura vegetal, maior o número de casos, dessa forma foi necessário inverter os valores máximo e mínimo e o mínimo na lógica fuzzy.

Definidos os valores da regressão linear e realizada a normalização dos mapas com a lógica fuzzy, foi gerado o mapa final, selecionando a ferramenta raster calculator. Criou-se uma fórmula com os valores encontrados para cada variável na regressão linear e multiplicando-os pelos referidos mapas e assim gerando um mapa final de suscetibilidade para casos de dengue, em degradê de cinza, com o cinza claro representando os bairros com menor suscetibilidade e o cinza escuro aqueles mais suscetíveis para os casos de dengue.

As técnicas de geoprocessamento e a representação em mapas possibilitam a compreensão detalhada das características geográficas, acerca das condições de saúde da população, contribuindo para o desenvolvimento de hipóteses sobre a situação de risco e de vulnerabilidade em saúde pública, a fim de colaborar nas ações de intervenção, tendo em vista os locais mais vulneráveis a doença, conforme os princípios da geografia da saúde (Santana 2014, Flauzino et. al., 2009, Penso e Périco, 2017, Brasil, 2006b, Brasil 2007).

escala 1:50.000. Porto Alegre, UFRGS-IB-Centro de Ecologia. 


\section{Resultados e discussão}

Os resultados indicam que, em Porto Alegre, RS, a precipitação não influencia significativamente o número de fêmeas coletadas $(\mathrm{p}=0,3505)$. A relação da precipitação com $\mathrm{o}$ número de fêmeas é bem clara em regiões do país onde as estações climáticas não são bem definidas, por exemplo, estudos apontam associação entre os casos de dengue com a precipitação em unidades geográficas da Paraíba (Silva et. al., 2015, Almeida e Silva, 2017).

Em relação à associação entre a temperatura máxima nos seis meses amostrados e o número médio de fêmeas, os resultados apontam que a temperatura apresenta uma relação significativa $\left(\mathrm{p}=0,0027, \mathrm{R}^{2}=89,21 \%\right)$. Logo, o número de fêmeas coletadas eleva-se com o aumento da temperatura, seguindo a tendência dos resultados dos estudos de Câmara et al. (2009) e Ribeiro et al. (2006), e diferenciando-se, daquilo apresentado por Almeida e Silva (2017).

Segundo Mendonça (2003, p. 215) “A distribuição dos mosquitos, a frequência de suas picadas e o período de incubação do vírus são afetados pela temperatura; com uma temperatura de $27^{\circ} \mathrm{C}$, por exemplo, o período de incubação é de 10 dias, com $37^{\circ} \mathrm{C}$ é de 7 dias".

A incidência de casos de dengue também flutua com as condições climáticas e está associada com o aumento da temperatura e pluviosidade, condições que favorecem para o aumento do número de criadouros disponíveis e também e para o desenvolvimento do vetor (Depradine e Lovell, 2004).

Mendonça et. al., (2009) discorrem acerca do impacto das alterações do tempo e do clima no que tange a proliferação dos vetores de doenças transmissíveis, citando o exemplo clássico do fenômeno El Nino quando "a intensidade e o regime pluviométrico e térmico observados no biênio 1997 e 1998, por exemplo, incidiram diretamente na ocorrência de epidemias de dengue na Ásia e de malária no Quênia, país que tinha relatado seu último caso da doença em 1952" (p.259). Porém, as análises demostraram que a pluviosidade não apresentou uma relação significativa, ao contrário da temperatura.

Estudos semelhantes como o de Câmara et al. (2009) no Rio de Janeiro, RJ, demostrou que as temperaturas dos primeiros trimestres do período de 1986-2003, especialmente as mínimas, mostraram-se significativamente mais altas nos anos em que as epidemias de dengue tiveram início na cidade.

Mondini e Neto (2007) em São José do Rio Preto, SP apontam a influência de fatores ambientais, principalmente a temperatura, que se faz marcante na dinâmica populacional da espécie em questão. Em climas caracterizados pelas variações sazonais, poderá haver períodos favoráveis à intensa proliferação do mosquito.

Essas flutuações fazem com que as epidemias manifestem-se em épocas até certo ponto previsíveis. Araújo e Nunes (2005) em São Luís do Maranhão, MA, observaram, durante o verão, um aumento circunstancial em decorrência de altas temperaturas, favorecendo assim o aumento dos índices de infestação e da densidade vetorial.

Com relação à pluviosidade, Moore (1985), analisando a infestação de Aedes aegypti em relação a dados climáticos, mostrou que a temperatura não foi um bom indicador, porém o volume e o número de dias com chuva podem constituir preditores úteis de sua abundância. Rebêlo et. al. (1999), também encontraram ligação direta entre a pluviosidade e a proliferação do mosquito.

Os resultados obtidos neste trabalho não encontraram relação significativa com a pluviosidade, mas sim com a temperatura. Tem-se por hipótese que isso esteja associado com as características subtropicais características da região sul do País, que contempla estações bem definidas e regime de chuvas estável, fazendo com a pluviosidade não exerça tanta influência sobre a proliferação do mosquito quanto a temperatura.

Ocorreram diferenças nas médias de fêmeas coletadas por mês de amostragem $(\mathrm{H}=$ $91,04, \mathrm{p}<0,0001)$. Foram observadas as seguintes médias nos meses: janeiro $(X=99,1)$, fevereiro $(122,5)$, março $(94,5)$ e abril $(93,6)$ essas médias não diferenciaram entre si, mas diferenciam significativamente dos meses de junho $(13,5)$ e julho $(3,3)$. O mês de maio $(53,5)$ difere de julho $(13,5)$, mas não dos demais. Junho $(13,5)$ não difere de julho. Considerando a temperatura necessária para que o mosquito complete seu ciclo de vida, uma redução nos meses mais frios do ano já era esperada, mesmo assim é estranho a amostragem de fêmeas adultas até julho.

A umidade do ar esteve associada com os casos de dengue nos estudos de Magalhães (2015), Almeida e Silva (2017) e Donalisio e Glasser (2002), neste último sobre a associação com a longevidade do vetor, indicando que pode favorecer a fêmea a participar de mais de um ciclo de replicação do vírus, possibilitando a contaminação de um maior número de indivíduos.

A análise de regressão múltipla $(\mathrm{F}=4,16$; $\left.p=0,0118 ; R^{2}=62,47 \%\right)$ utilizando como variável dependente o número de casos, indicou influência significativa das variáveis número de fêmeas $(\mathrm{p}=$ 
0,$\left.0420 ; \mathrm{R}^{2}=3,4 \%\right)$ e uso antrópico $\left(\mathrm{p}=0,0003, \mathrm{R}^{2}\right.$ $=10,89 \%$ ). Os coeficientes de determinação foram baixos para todas as variáveis, indicando que nenhum dos fatores testados influencia significativamente, de forma individual, o número de casos.

Tabela 1. Bairros, área (em ha) e percentual (\%) das classes avaliadas. A classe "Antrópico" abrange todos os demais usos e coberturas do solo. Fonte: Modificado de Caldas et al.,2015.

\begin{tabular}{|c|c|c|c|c|c|c|c|c|c|c|c|c|c|}
\hline \multirow{3}{*}{$\begin{array}{c}\text { Nome dos } \\
\text { bairros }\end{array}$} & \multirow{3}{*}{$\mathrm{Nr}$} & \multicolumn{2}{|c|}{ Água } & \multicolumn{2}{|c|}{ Arbóreo } & \multicolumn{2}{|c|}{ Arbustivo } & \multicolumn{2}{|c|}{ Herbáceo } & \multicolumn{2}{|c|}{ Antrópico } & \multicolumn{2}{|c|}{ Total } \\
\hline & & \multicolumn{12}{|c|}{ ÁREA } \\
\hline & & ha & $\%$ & ha & $\%$ & Há & $\%$ & ha & $\%$ & ha & $\%$ & ha & $(\%)$ \\
\hline Azenha & 1 & 1,3 & 1,0 & 1,8 & 1,4 & 0,0 & 0,0 & 2,2 & 1,8 & 121,7 & 95,8 & 127,0 & 100,0 \\
\hline Bom Jesus & 2 & 0,0 & 0,0 & 0,0 & 0,0 & 0,0 & 0,0 & 0,8 & 0,4 & 193,6 & 99,6 & 194,4 & 100,0 \\
\hline Cavalhada & 3 & 0,1 & 0,0 & 31,6 & 8,8 & 10,0 & 2,8 & 17,8 & 5,0 & 298,1 & 83,3 & 357,6 & 100,0 \\
\hline & 4 & 0,0 & 0,0 & 19,5 & 6,7 & 3,4 & 1,2 & 29,5 & 10,2 & 236,9 & 81,9 & 289,3 & 100,0 \\
\hline Chác. Pedras & 5 & 0,0 & 0,0 & 1,2 & 1,1 & 0,0 & 0,0 & 4,7 & 4,4 & 102,2 & 94,5 & 108,1 & 100,0 \\
\hline Cidade Baixa & 6 & 0,0 & 0,0 & 0,5 & 0,6 & 0,0 & 0,0 & 0,3 & 0,4 & 83,1 & 99,1 & 83,9 & 100,0 \\
\hline Farroupilha & 7 & 1,7 & 2,9 & 21,4 & 35,8 & 0,0 & 0,0 & 9,5 & 15,9 & 27,2 & 45,4 & 59,8 & 100,0 \\
\hline Glória & 8 & 0,0 & 0,0 & 6,7 & 6,3 & 0,0 & 0,0 & 1,0 & 0,9 & 99,4 & 92,8 & 107,1 & 100,0 \\
\hline Jardim Carvalho & 9 & 1,9 & 0,6 & 62,8 & 20,3 & 7,7 & 2,5 & 26,7 & 8,6 & 210,5 & 68,0 & 309,7 & 100,0 \\
\hline Jardim do Salso & 10 & 1,5 & 1,6 & 5,8 & 6,3 & 13,3 & 14,3 & 19,9 & 21,4 & 52,8 & 56,6 & 93,3 & 100,0 \\
\hline Mario Quintana & 11 & 0,0 & 0,0 & 32,6 & 4,9 & 137,7 & 20,6 & 244,6 & 36,5 & 254,4 & 38,0 & 669,3 & 100,0 \\
\hline Medianeira & 12 & 0,0 & 0,0 & 0,3 & 0,2 & 0,0 & 0,0 & 3,6 & 2,4 & 150,4 & 97,4 & 154,4 & 100,0 \\
\hline Menino Deus & 13 & 1,8 & 0,8 & 3,9 & 1,8 & 0,0 & 0,0 & 8,4 & 3,8 & 206,7 & 93,6 & 220,9 & 100,0 \\
\hline Nonoai & 14 & 0,0 & 0,0 & 111,3 & 24,7 & 22,5 & 5,0 & 59,5 & 13,2 & 258,1 & 57,2 & 451,4 & 100,0 \\
\hline Partenon & 15 & 0,1 & 0,0 & 25,2 & 4,4 & 8,5 & 1,5 & 48,8 & 8,5 & 490,4 & 85,6 & 572,9 & 100,0 \\
\hline Passo da Areia & 16 & 0,0 & 0,0 & 15,5 & 6,2 & 1,1 & 0,4 & 21,6 & 8,7 & 211,1 & 84,7 & 249,3 & 100,0 \\
\hline Passo das Pedras & 17 & 0,3 & 0,1 & 12,9 & 5,6 & 29,0 & 12,7 & 47,6 & 20,8 & 138,5 & 60,7 & 228,3 & 100,0 \\
\hline Santa Tereza & 18 & 0,0 & 0,0 & 54,3 & 11,2 & 12,8 & 2,7 & 36,4 & 7,5 & 380,6 & 78,6 & 484,1 & 100,0 \\
\hline Santo Antônio & 19 & 0,0 & 0,0 & 7,2 & 5,3 & 0,0 & 0,0 & 1,5 & 1,1 & 128,0 & 93,6 & 136,7 & 100,0 \\
\hline São José & 20 & 0,0 & 0,0 & 3,8 & 1,8 & 0,0 & 0,0 & 5,3 & 2,5 & 205,2 & 95,7 & 214,3 & 100,0 \\
\hline Três Figueiras & 21 & 0,0 & 0,0 & 2,6 & 1,9 & 13,3 & 10,0 & 8,5 & 6,4 & 109,0 & 81,7 & 133,4 & 100,0 \\
\hline V. João Pessoa & 22 & 0,6 & 0,7 & 0,0 & 0,0 & 0,0 & 0,0 & 1,2 & 1,3 & 87,6 & 98,0 & 89,3 & 100,0 \\
\hline \multicolumn{2}{|l|}{ TOTAL } & 9,2 & 0,2 & 421,0 & $\mathbf{7 , 9}$ & 259,4 & 4,9 & $\mathbf{5 9 9 , 5}$ & 11,2 & 4045,2 & $\mathbf{7 5 , 8}$ & $\mathbf{5 3 3 4 , 4}$ & 100,0 \\
\hline
\end{tabular}

A análise de regressão múltipla $(\mathrm{F}=4,40$; $\left.\mathrm{p}=0,010 ; \mathrm{R}^{2}=57,91 \%\right)$ utilizando como variável dependente o número de fêmeas, indicou influência significativa do uso antrópico $\left(\mathrm{p}=0,0003, \mathrm{R}^{2}=\right.$ $38,63 \%$ ).

Os dados de uso e cobertura da terra foram obtidos para cada bairro amostrado e, separados nas seguintes classes: cobertura vegetal (arbóreo, arbustivo e herbáceo), água e uso antrópico (tabela 1 e Figura 5).
Flauzino et al. (2009) consideram "importante o foco dos estudos com dados secundários tanto na agregação de unidades espaciais quanto nas características ambientais [...] diferenciando-se dos inquéritos sorológicos, que abordam especialmente as características dos indivíduos, sem preocupar-se com o nível ambiental" (p. 460). 


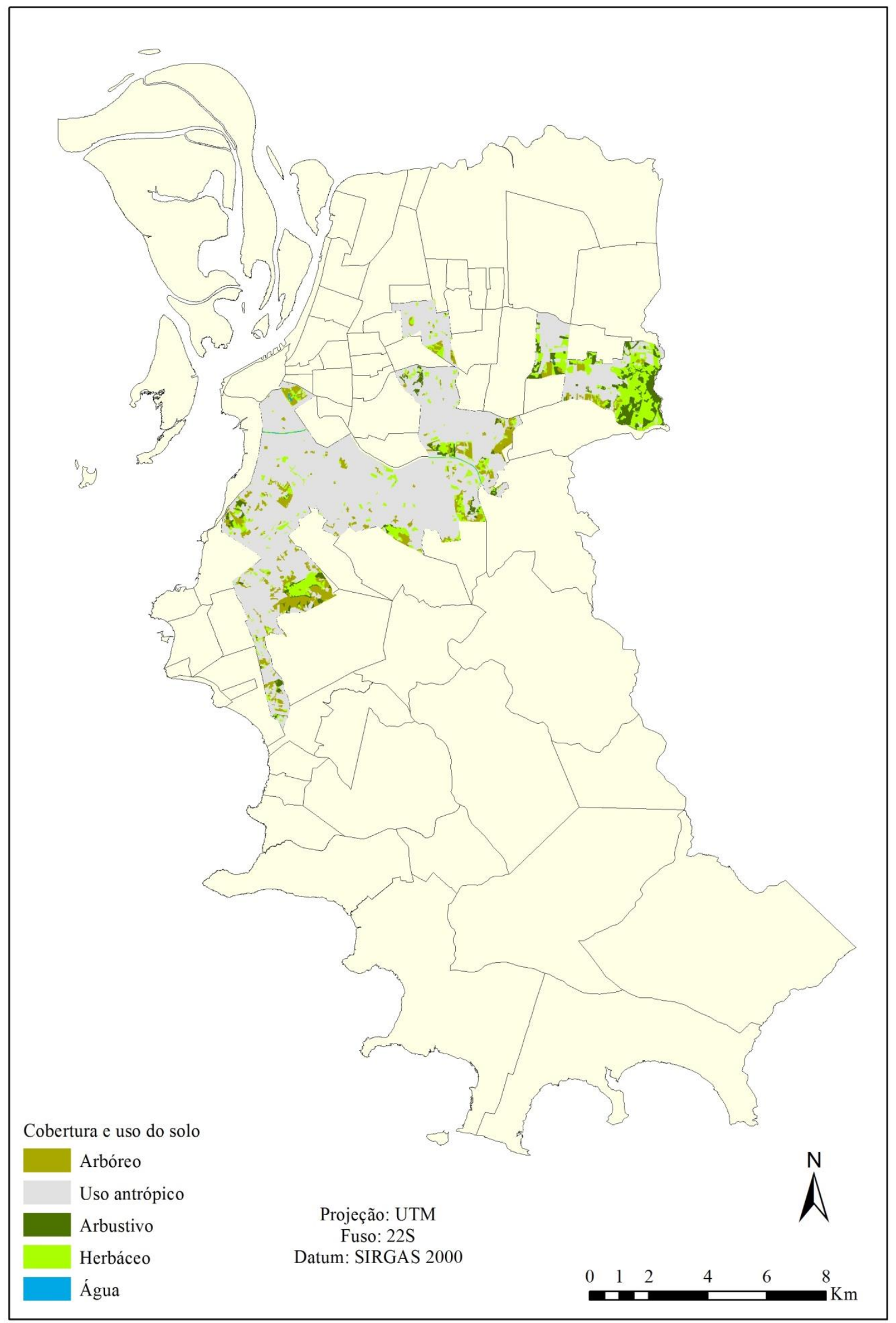

Figura 5. Mapa de uso e cobertura do solo, nos bairros analisados em Porto Alegre, RS. 
A expansão das áreas de ocorrência de dengue no mundo e no Brasil está associada tanto à urbanização, sem a devida estrutura de saneamento, quanto a fatores socioeconômicos.

Em um estudo de revisão aponta-se que "na análise para fatores de risco, ficou evidente que os agregados de quarteirões que apresentaram alta densidade populacional, presença de grandes centros comerciais e educacionais e presença de vetor foram os de maior risco para a doença" (Martinez 2003 apud Flauzino et. al. 2009, p.459), o mesmo estudo identifica também locais de risco nas unidades geográficas que integram hotéis ou alojamentos para turistas (Martinez 2003 apud Flauzino et. al. 2009).

Em 2010 houve o primeiro caso de dengue autóctone na cidade de Porto Alegre e desde então tem ocorrido uma tendência de crescimento/ascensão: em 2013 foram confirmados 150 casos configurando-se como a maior incidência de dengue na cidade ${ }^{3(\text { acima) }}$. Nem sempre a presença de fêmeas está relacionada com a maior incidência de casos (Figura 6). Isso se deve, em parte, porque os fatores de contaminação das fêmeas também dependem das características ambientais.

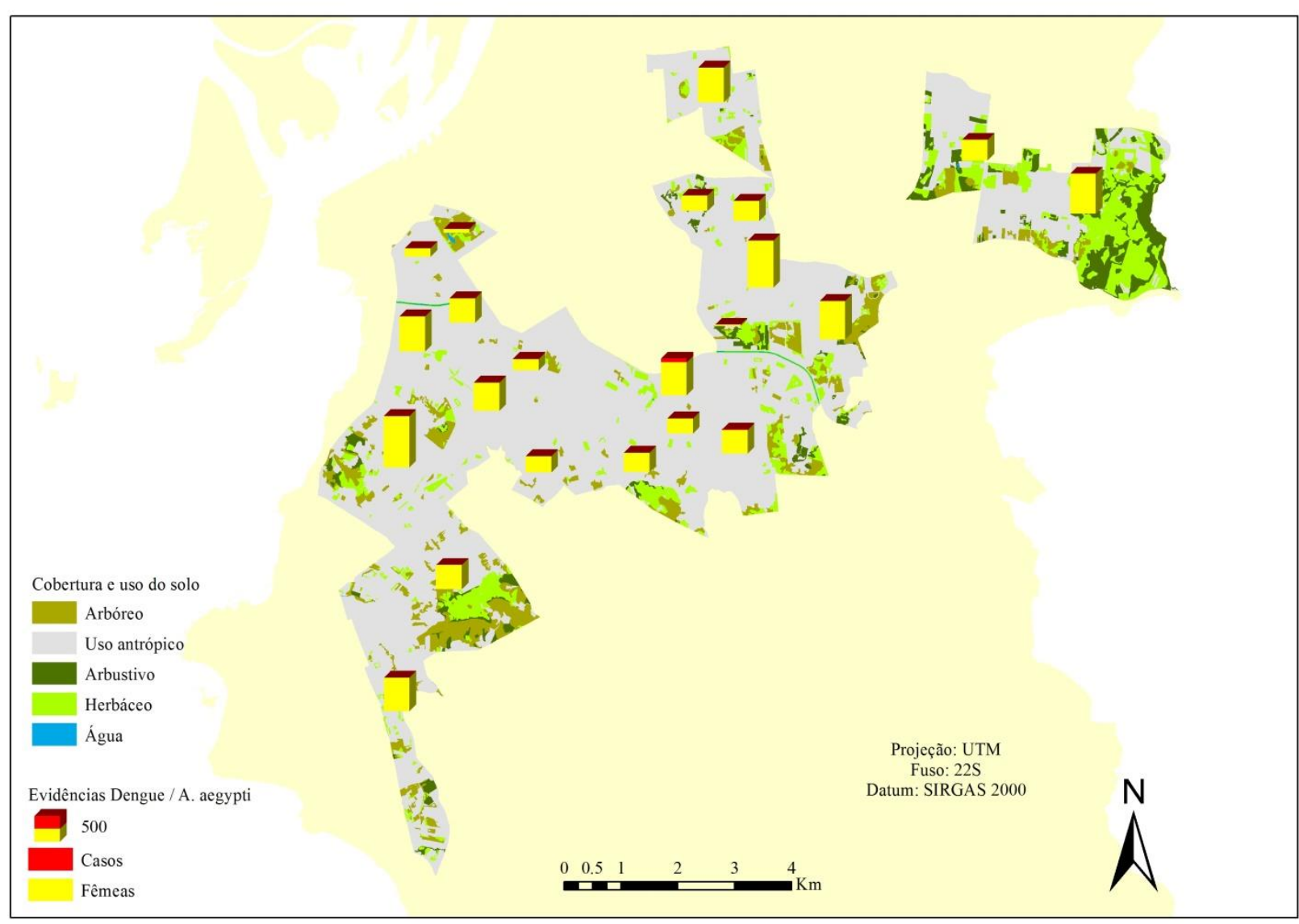

Figura 6. Número absoluto de fêmeas de Aedes aegypti coletadas e casos de dengue em relação ao uso e cobertura do solo, Porto Alegre, RS.

O nível socioeconômico também não se mostrou um fator significativo, embora haja uma tendência de aumento do número de fêmeas coletadas à medida que diminui a renda média do bairro amostrado $\left(p=0,0819 ; R^{2}=14,36 \%\right)$.

Mondini e Neto (2007) em estudos realizados em São José do Rio Preto, SP, observaram, utilizando análise de componentes principais, que o fator socioeconômico seria responsável por $87 \%$ da variação total nos anos de 1994-1995.

Apontou-se a idade entre os 15 aos 49 anos como fator de risco para a doença, sem diferença significativa entre os sexos (Gonçalves Neto, Rebelo, 2004), embora existam evidências de que a faixa etária entre 20 a 29 anos (Marçal Júnior, Santos, 2004) e de 20 a 39 (Ribeiro et. al., 2006) também possam ser fatores de vulnerabilidade.

Os mapas de suscetibilidade são importantes para uma compreensão mais integral dos locais mais vulneráveis a determinadas condições ou situações de saúde, tendo em vista as causas multifatoriais relacionadas (Brasil, 2006b; Brasil 2007; Santana 2014; Penso, Périco, 2017).

Os bairros mais suscetíveis à dengue em Porto Alegre são: Azenha, Bom Jesus, Chácara das 
Pedras, Cidade Baixa, Medianeira, Menino Deus, Santo Antônio, São José e Vila João Pessoa (Figura 7). Este resultado possibilita o embasamento de ações de conscientização sobre práticas educativas relacionadas à prevenção do mosquito, principalmente nos locais com maior predisposição ao vetor.

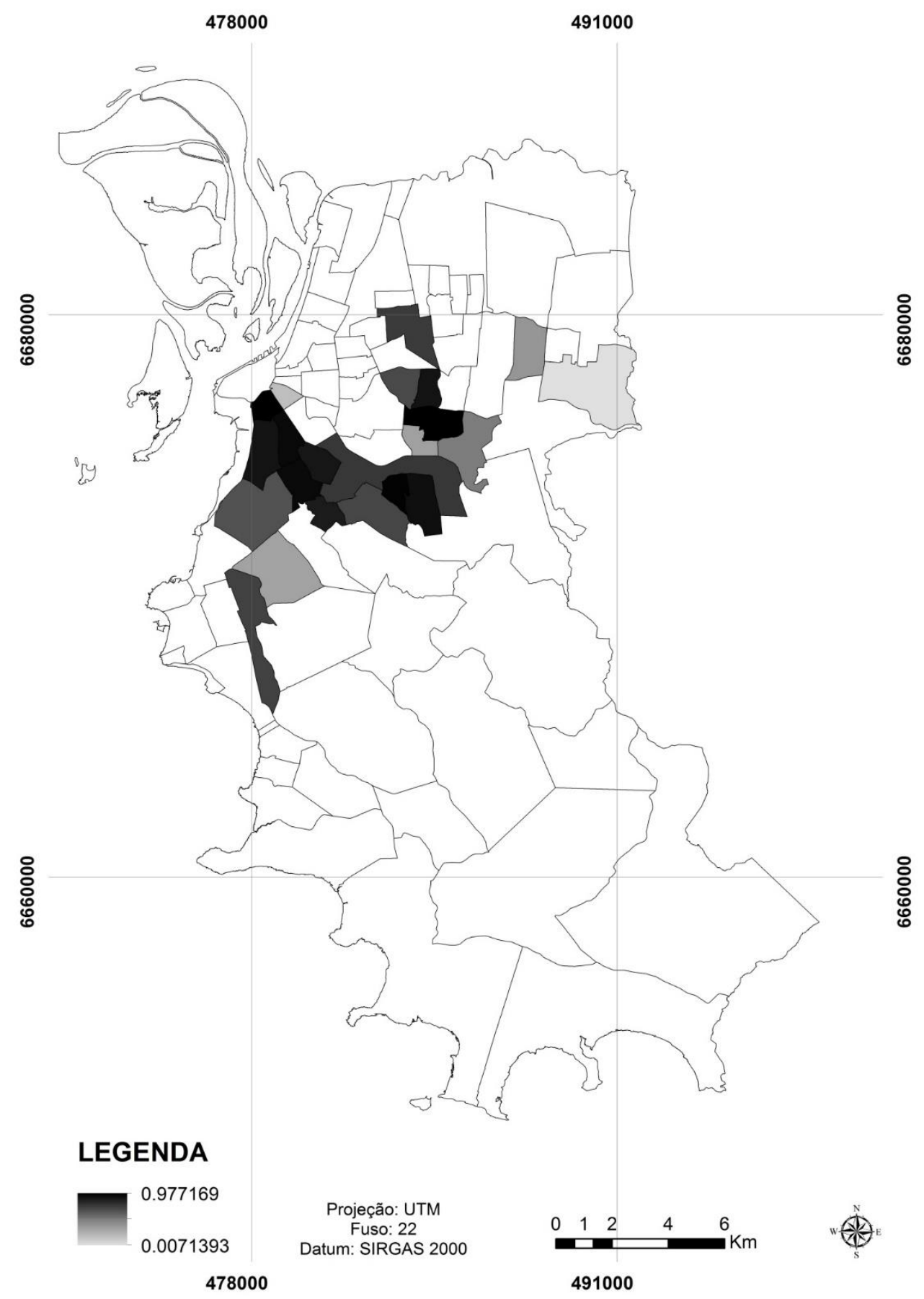

Figura 7. Mapa de suscetibilidade para dengue, nos bairros amostrados em Porto Alegre, RS.

O período de 1950 e 1960 ilustrou o desenvolvimento de estratégias que resultaram na eliminação do transmissor da febre amarela no Brasil, doença epidêmica no período. As ações desenvolvidas naquele momento foram perpassadas pelo comprometimento das autoridades sanitárias e da população.
Segundo Mendonça et. al. (2009) "com o sucesso obtido naquela campanha, parece ter ocorrido uma despreocupação quanto ao controle do mosquito, principalmente numa época favorável à sua disseminação em decorrência da urbanização intensa e desorganizada no Brasil" (p.259). 


\section{Conclusão}

Em cidades brasileiras, que apresentam a sazonalidade bem definida, aponta-se a escassez de estudos que relacionam aspectos da paisagem, como o uso e cobertura do solo, com os casos de dengue. Os dados indicam que embora as variáveis de cobertura do solo não expliquem totalmente os casos registrados em Porto Alegre, o processo de urbanização apresenta influência significativa sobre o número de fêmeas, e do número casos

Os bairros que apresentaram menos cobertura vegetal e maior área de uso antrópico (edificações) foram os mais suscetíveis ao aparecimento da doença. Ao contrário da literatura, que cita a pluviosidade bem distribuída ao longo dos anos concomitante a temperaturas elevadas, no presente estudo os principais fatores são: a temperatura relacionada diretamente a eclosão dos adultos, a variedade de opções de criadouros para desenvolvimento das larvas e a urbanização.

A hipótese levantada pelo presente estudo é de que quanto menor a taxa de urbanização dos bairros e maior a disponibilidade de áreas verdes, que tendem apresentar menos criadouros aos vetores, menor a tendência para o número de casos de dengue, provavelmente devido a manutenção de inimigos naturais das larvas e adultos

Os resultados indicam que o método de armadilhas é adequado para um levantamento dos

\section{Agradecimentos}

À Coordenação de Aperfeiçoamento de Pessoal de Nível Superior (CAPES), pela concessão de bolsa de doutorado à primeira autora. Ao Engenheiro Ambiental Daniel Martins dos Santos pelo auxílio na confecção dos mapas.

\section{Referências}

Almeida, C. A. P., Silva, R. M., 2017. Modelagem espacial dos casos de dengue e variáveis socioambientais em João Pessoa, Cabedelo e Bayeux, Paraíba. Revista Brasileira de Geografia Física 10, 1455-1470.

Araújo, R. R., Nunes, J. S. A., 2005. Relações geográficas entre o clima e a incidência de dengue na cidade de São Luís - MA. Ciências Humanas em Revista 3, 86-97.

Baroni, C. J., Oliveira T. B., 2009. Aspectos epidemiológicos da febre clássica da dengue, em Giruá - RS. Revista Brasileira de Análises Clínicas 41, 289-293.

Brasil, 2006a. Programa Nacional de Controle da Dengue: amparo legal à execução das ações de campo - imóveis fechados, abandonados ou com acesso não permitido pelo morador. casos da doença em regiões urbanas. A precipitação não influenciou o número de fêmeas encontradas, mas a elevação da temperatura está diretamente correlaciona a um aumento no número de fêmeas.

Nos 22 bairros analisados o número de casos autóctones de dengue apresenta influência significativa do número de fêmeas presentes, da cobertura vegetal e do uso antrópico do solo. O parcelamento ordenado das classes de uso do solo podem ser fatores determinantes na presença de casos de dengue nos bairros avaliados.

Em suma, considerando variáveis multifatoriais, os resultados do estudo responderam ao problema proposto, o desenvolvimento de um mapa de suscetibilidade para a dengue no município de Porto Alegre, indicando-se que entre os bairros analisados, os que são mais predispostos ao vetor são: Azenha, Bom Jesus, Chácara das Pedras, Cidade Baixa, Medianeira, Menino Deus, Santo Antônio, São José e Vila João Pessoa.

Este resultado é relevante para uma compreensão integral dos locais mais vulneráveis a doença e, embora este estudo não seja conclusivo, é relevante para o embasamento de ações urgentes de prevenção à doença e combate ao vetor nos locais mais suscetíveis.

Ministério da Saúde, Secretaria de Vigilância em Saúde 2. ed.

Brasil, 2006b. Abordagens espaciais na saúde pública. Ministério da Saúde 1, Brasília.

Brasil, 2007. Introdução à Estatística Espacial para a Saúde Pública. Ministério da Saúde 1, Brasília.

Brasil, 2017. Ministério da Saúde. Portaria $N^{\circ}$ 1.682, de 30 de Julho de 2017 - Declara o encerramento da Emergência em Saúde Pública de importância Nacional (ESPIN) por alteração do padrão de ocorrência de microcefalias no Brasil e desativa o Centro de Operações de Emergências em Saúde Pública (COES).

Brasil, 2009. Diretrizes nacionais para prevenção e controle de epidemias de dengue. Ministério da Saúde 1, 160 p. (Série A. Normas e Manuais Técnicos).

Brasil, 2015. Ministério da Saúde. Portaria $\mathrm{n}^{\circ}$ 1.813, de 11 de novembro de 2015 - Declara Emergência em Saúde Pública de importância Nacional (ESPIN) por alteração do padrão de ocorrência de microcefalias no Brasil.

Caldas, E., Santos, D., Gallas, M., Silveira, E.F., Périco, E. 2015. Influência de determinantes ambientais e socioeconômicos nos casos de dengue na cidade de Porto Alegre, RS Anais 
XVII Simpósio Brasileiro de Sensoriamento Remoto - SBSR, João Pessoa-PB, Brasil, 25 a 29 de abril de 2015, INPE.

Câmara, F. P., Gomes, A. F., Santos, G. T., Câmara, D. C. P., 2009. Clima e epidemias de dengue no Estado do Rio de Janeiro. Revista da Sociedade Brasileira de Medicina Tropical 42, 137-140.

Costa, A. I. P., Natal D., 1998. Distribuição espacial da dengue e determinantes socioeconômicos em localidade urbana no sudeste do Brasil. Revista Saúde Pública 32, 232-236.

Depradine, C. A., Lovell, E. H., 2004. Climatological variables and the incidence of dengue fever in Barbados. International Journal of Environmental Health Research 14, 429-441.

Donalisio, M. R., Glasser, C. M., 2002. Vigilância entomológica e controle de vetores da dengue. Revista Brasileira de Epidemiologia 5, 259279.

Flauzino, R. F., Souza-Santos, R., Oliveira, R. M., 2009. Dengue, geoprocessamento e indicadores socioeconômicos e ambientais: um estudo de revisão. Revista Panamericana de Salud Pública 25, 456-461.

Forattini, O. P., 1992. Ecologia, epidemiologia e sociedade. São Paulo: Artes Médicas. 529p.

Goncalves neto, V., Rebelo, J. 2004. Aspectos epidemiológicos do dengue no município de São Luís, Maranhão, Brasil, 19972002. Cadernos de Saúde Pública 20, 14271431.

Honório, N. A., Oliveira, R. L., 2001. Freqüência de larvas e pupas de Aedes aegypti e Aedes albopictus em armadilhas, Brasil. Revista Saúde Pública 35, 385-391.

Instituto Brasileiro de Geografia e Estatística IBGE, 2017. Cidades. Disponível em: <http://cidades.ibge.gov.br/xtras/perfil.php?co $\mathrm{dmun}=431490>$. Acesso em: 9 de março de 2018.

Magalhães, G. B., 2015. Comportamento Espaçotemporal da Dengue e sua Relação com os Elementos Atmosféricos e Socioeconômicos em Fortaleza/CE. Tese (Doutorado). Fortaleza, UFC.

Marçal Junior, O., Santos, A. 2004. infestação por Aedes aegypti (diptera: culicidae) e incidência do dengue no espaço urbano: um estudo de caso. Caminhos de Geografia 5, n.13, 241-251.
Mendonça, F. A., Veiga e Souza, A., Dutra, D. A., 2009. Saúde pública, urbanização e dengue no Brasil. Sociedade \& Natureza 21, 257-269.

Mendonça, F. Aquecimento global e saúde: uma perspectiva geográfica - notas introdutórias. Revista Terra Livre 20, 205-221.

Mondini, A., Neto, F. C., 2007. Variáveis socioeconômicas e a transmissão da dengue. Revista Saúde Pública 41, 923-930.

Moore, C. G., 1985. Predicting Aedes aegypti abundance from climatological data. Florida Medical Entomology Laboratory 2, 223-233.

Penso, J. M., Périco, E., 2016. Análise espaçotemporal da mortalidade por diabetes mellitus no Rio Grande do Sul. Revista Brasileira de Geografia Física 09, 1836-1848.

Périco, E., Cemin, G. 2006. Caracterização da paisagem do município de Arvorezinha, RS, com ênfase na dinâmica dos fragmentos florestais, pormeio de sistemas de informações geográficas (SIGs).Scientia Forestalis 70, 0921.

Rebêlo, J. M. M., Costa, J. M. L., Silva, F. S., Pereira, Y. N. O., Silva, J. M., 1999. Distribuição de Aedes aegypti e do dengue no Estado do Maranhão, Brasil. Caderno de Saúde Pública 15, 477-486.

Ribeiro, A. F., Marques, G. R. A. M., Voltolini, J. C., Condino, M. L. F., 2006. Associação entre incidência de dengue e variáveis climáticas. Revista Saúde Pública 40, 671-676.

Santana, P., 2014. Introdução à geografia da saúde: território, saúde e bem estar. Universidade de Coimbra. Coimbra University Press 1, 192p.

Silva, A. M., Silva, R. M., Almeida, C. A. P, Chaves, J. J. S., 2015. Modelagem Geoestatística dos Casos de Dengue e da Variação Termopluviométrica em João Pessoa, Brasil. Revista Sociedade e Natureza 27, 157169.

Tauil, P. L., 2001, Urbanização e ecologia do dengue. Cadernos de Saúde Pública 17, 99-102.

Valdés, G. L., Mizhrahi, J. V., Guzmán, M. G., 2002. Impacto económico de la epidemia de dengue 2 en Santiago de Cuba, 1997. Revista Cubana de Medicina Tropical 54, 220-227. 